\title{
What's in SWAP? Abundance of the principal constituents in a soluble extract of Schistosoma mansoni revealed by shotgun proteomics
}

\author{
Leandro Xavier Neves ${ }^{1}$, Ananda Lima Sanson ${ }^{1}$, R. Alan Wilson² and William Castro-Borges ${ }^{1 *}$
}

\begin{abstract}
Background: The soluble antigen preparation of adult schistosomes (SWAP) has often been used to probe host responses against these blood-dwelling parasites. Despite its long-established use there is limited knowledge about its composition. The information we provide here on the molecular composition of SWAP may contribute as a guide for a rational selection of antigenic targets.
\end{abstract}

Methods: Label-free quantitative shotgun proteomics was employed to determine the composition and abundance of SWAP constituents. Briefly, paired adult Schistosoma mansoni worms were sonicated in PBS pH 7.2 and ultracentrifuged for recovery of the soluble supernatant. An aliquot was subjected to trypsin digestion. Resulting peptides were separated under ultra-high performance liquid chromatography and analysed using an orbitrap mass spectrometer. Spectral data were interrogated using SequestHT against an in-house S. mansoni database. Proteins were quantified by recording the mean area under curve obtained for up to three most intense detected peptides. Proteins within the $90^{\text {th }}$ percentile of the total SWAP mass were categorized according to their sub-cellular/tissue location.

Results: In this work we expanded significantly the list of known SWAP constituents. Through application of stringent criteria, a total of 633 proteins were quantitatively identified. Only 18 proteins account to $50 \%$ of the total SWAP mass as revealed by their cumulative abundance. Among them, none is predicted as a secreted molecule reinforcing the point that SWAP is dominated by cytosolic and cytoskeletal proteins. In contrast, only $3 \%$ of the mass comprised proteins proposed to function at the host-parasite interfaces (tegument and gut), which could conceivably represent vulnerable targets of a protective immune response. Paradoxically, at least 11 SWAP proteins, comprising 25\% of its mass, have been tested as vaccine candidates.

Conclusions: Our data suggest that use of SWAP to probe host responses has greatest value for diagnostic purposes or assessing intensity of infection. However, the preparation is of limited utility as an antigen source for investigating host responses to proteins expressed at or secreted from worm-host interfaces. The data also pose the question as to why vaccination with SWAP, containing so many proposed vaccine candidates, has no additive or even synergistic effects on the induction of protection.

Keywords: Schistosoma mansoni, Soluble proteome, Quantitative shotgun proteomics, Vaccine candidates

\footnotetext{
* Correspondence: wborges@iceb.ufop.br

'Laboratório de Enzimologia e Proteômica, Instituto de Ciências Exatas e Biológicas, Universidade Federal de Ouro Preto, Ouro Preto, Minas Gerais, Brazil

Full list of author information is available at the end of the article
} 


\section{Background}

SWAP (or SWA) is a soluble adult worm antigen preparation universally used by immunologists to probe host responses to schistosome infections. It is essentially a Tris- $\mathrm{HCl}$ or PBS-based extract of mixed male and female worms variously prepared by homogenization, sonication or freeze/thaw (or a combination of these) followed by a high-speed centrifugation to remove particulate material. It yields a solution typically containing 3-5 mg protein $/ \mathrm{mL}$ which can be stabilized by adding protease inhibitor cocktail for ELISA and Western blotting or used without inhibitors for cell-stimulation assays. The first report on its use appears to be from 1977 [1] and 38 years later it is still a standard preparation. In one recent publication SWAP was used to investigate human anti-fecundity immunity to Schistosoma haematobium using antibody isotype ELISAs as the assay of reactivity [2]. In a second study, a version of SWAP supplemented by proteins soluble in chaotropic agents and mild detergents provided the material for an immunoproteomic analysis of human antibody responses, using 2D western blotting to select reactive constituents [3].

In spite of this long-established usage very little is known about its precise composition. The first proteomic study of S. mansoni, using 2D-gel technology and peptide-mass fingerprinting, provided a comparison of soluble preparations from four different life-cycle stages, among them adult worms [4]. The 40 most abundant spots from 2D gel comprised glycolytic enzymes (e.g enolase, aldolase, GAPDH, TPI), cytoskeletal proteins (actin, myosin light chain, tropomyosin), chaperones (Hsp-70, 14-3-3), stress response proteins (superoxide dismutase, GST-28) and calcium-binding proteins (calponin and EF-hand Sm21.7). However, no information was provided on relative abundance of the components. Peptide mass fingerprinting was superseded more than a decade ago by peptide fragmentation as a more accurate means of protein identification. Whilst gel technology is still useful in some applications, for compositional studies quantitative shotgun proteomics is now the method of choice [5]. Furthermore, the advances in mass spectrometer design have increased the sensitivity of peptide detection by orders of magnitude $[6,7]$.

Motivated by a feeling that immunologists really ought to know exactly what they are probing their cell or antibody responses with when they use SWAP, we describe here an analysis of its composition using state-of-the-art instrumentation in conjunction with software for protein identification and quantitation. We have shown that at least $80 \%$ of the identified molecules are primarily of intracellular origin whereas only $3 \%$ represent signature proteins derived from the tegument and gastrodermis, the major host-parasite interfaces. The information we provide on the composition and abundance of SWAP constituents should better enable immunologists to appreciate exactly what antigenic reactivities they are monitoring.

\section{Methods}

\section{Ethics statement}

The protocol for maintenance of the $S$. mansoni life cycle was reviewed and approved by the local ethics committee on animal experimentation, Comissão de Ética no Uso de Animais (CEUA), Universidade Federal de Ouro Preto (UFOP), and received the protocol no. 2011/55.

\section{SWAP or (SWA) - soluble worm antigen preparation}

Approximately 100 adult paired worms were sonicated in $1 \mathrm{~mL}$ PBS pH 7.2 containing $1 \mathrm{x}$ Protease Inhibitor Cocktail (Sigma Aldrich, St. Louis, USA). The homogenate was clarified by centrifugation at 40,000 $\times \mathrm{g}$, for $2.5 \mathrm{~h}$, at $4{ }^{\circ} \mathrm{C}$ followed by recovery of the supernatant containing soluble antigens. The protein content was determined using PIERCE ${ }^{\mathrm{Ts}}$ BCA Protein Assay Kit (Thermo Scientific, Rockford, USA).

\section{In solution digestion and mass spectrometry analysis}

Proteins present in a $50 \mu \mathrm{g}$ aliquot of SWAP were first reduced using $2 \mathrm{mM}$ dithiothreitol (Sigma Aldrich) at $56{ }^{\circ} \mathrm{C}$, for $15 \mathrm{~min}$, in $100 \mathrm{mM}$ ammonium bicarbonate. After cooling at room temperature, proteins were then alkylated in $4.5 \mathrm{mM}$ iodoacetamide (Sigma Aldrich) for $15 \mathrm{~min}$ in the dark. The sample was then diluted 2.5 times using ultra-pure water and the ammonium bicarbonate concentration adjusted to $100 \mathrm{mM}$. Protein digestion was carried out at $37{ }^{\circ} \mathrm{C}$ for $16 \mathrm{~h}$ using Sequencing Grade Modified Trypsin (Promega, Madison, USA) at a protein to enzyme ratio of 25:1. Trypsinolysis was terminated by sample acidification with $4 \%(v / v)$ ultra-pure glacial acetic acid (J.T. Baker, Center Valley, USA). Peptides were cleaned up by solid phase extraction using a Strata $\mathrm{C}_{18}$-E cartridge $(55 \mu \mathrm{m}$, Phenomenex, Macclesfield, UK).

\section{LC-MS/MS analyses}

Five $\mu \mathrm{g}$ of the peptide preparation were loaded onto a UltiMate 3000 UHPLC system (Thermo Scientific, Bremen, Germany) equipped with an Acclaim PepMap100 $\mathrm{C}_{18}$ Nano-Trap Column $(75 \mu \mathrm{m}$ i.d. $\times 2$ cm, $3 \mu \mathrm{m}, 100 \AA$; Thermo Scientific) in line with an Acclaim PepMap100 $\mathrm{C}_{18}$ RSLC $(75 \mu \mathrm{m}$ i.d. $\times 15 \mathrm{~cm}, 2 \mu \mathrm{m}, 100 \AA$ A; Thermo Scientific) capillary column. Trapped peptides were washed for $3 \mathrm{~min}$ in $2 \%(v / v)$ acetonitrile/0.05 \% $(v / v)$ trifluoracetic acid, at a flow rate of $5 \mu \mathrm{L} / \mathrm{min}$, before switching the flow to the capillary column. Peptide separation was carried out at $40{ }^{\circ} \mathrm{C}$ under a multi-step gradient using a combination of solvents A $(0.1 \%(v / v)$ 
formic acid), and B (80 \% ( $v / v)$ acetonitrile/0.1 \% ( $v / v)$ formic acid). The gradient started from $4 \%$ solvent $B$, increased to $30 \%$ over $180 \mathrm{~min}$ and reached $55 \%$ at $240 \mathrm{~min}$. This was followed by a sharp ramp to $90 \%$ solvent B over $10 \mathrm{~min}$, maintenance at $90 \%$ for an additional $10 \mathrm{~min}$ and finally a drop to $4 \%$ over $20 \mathrm{~min}$ to restore the initial condition. All UHPLC solvents were purchased from J.T. Baker ${ }^{\circ}$.

A nano UHPLC system interfaced with a Q-Exactive ${ }^{\mathrm{Tm}}$ instrument (Thermo Scientific) allowed the mass spectrometric analysis of eluting peptides. A Nanospray Flex Ion Source (Thermo Scientific) fitted with a stainless steel nano-bore emitter needle $(150 \mu \mathrm{m}$ o.d. $\times 30 \mu \mathrm{m}$ i.d., Proxeon, Thermo Scientific) was operated at $1.9 \mathrm{kV}$, in positive mode and capillary temperature set to $250{ }^{\circ} \mathrm{C}$. Survey scans were acquired at a resolution of 70,000 with maximum injection time of $100 \mathrm{~ms}$ and target value of $3 \times 10^{6}$ ions. Up to 12 most abundant isotope patterns scanned in the range of $300-2000 \mathrm{~m} / z$, exhibiting charge state $\geq 2$ were isolated over a window of $2 \mathrm{~m} / z$ before fragmentation via higher-energy collisional dissociation (HCD) with normalized collision energy of $30 \mathrm{~V}$. MS/MS spectra were acquired at a 17,500 resolution with maximum injection time of $150 \mathrm{~ms}$ and target value of $2 \times 10^{5}$ ions. Dynamic exclusion of ions was set to $60 \mathrm{~s}$.

\section{Data processing}

Mass spectral data were submitted to database search using Proteome Discoverer software v.1.4 (Thermo Scientific). Our workflow included the SequestHT search engine plus Event Detector and Precursor Ions Area Detector for label-free quantification. Searching parameters included: 1) enzyme, trypsin/P; 2) maximum missed cleavage sites $=2$; 3) carbamidomethyl (C);4) methionine oxidation and $\mathrm{N}$-terminal acetylation as dynamic modifications; 5) mass tolerance of $10 \mathrm{ppm}$ for parental ions and $0.1 \mathrm{Da}$ for MS/MS. Spectra were searched against a local Schistosoma mansoni database (10,773 sequences; 5,135,674 residues). Only the protein identities that matched to at least one high confidence peptide were considered.

\section{Protein categorization and bioinformatic analyses}

Proteins were categorized by sub-cellular or tissue location using data from the S. mansoni database, Uniprot, and Blast searches. Signature proteins, here defined as known constituents of the soluble, gut-secreted and tegument proteomes, were listed based on published literature [4, 8-11]. Signal peptides were predicted using SignalP v3.0.

\section{Statistical analysis}

Arbitrary values related to individual protein abundance were tested for normal distribution using the Shapiro-Wilk test by the GraphPad Prism version 5.00 for windows, GraphPad software, San Diego, California, USA.

\section{Results}

Shotgun analysis revealed a large number of constituents in SWAP

A total of 60,571 peptides, selected by the Q-Exactive for fragmentation, yielded approximately 1000 identities when the latest version of the $S$. mansoni gene predictions was searched using Proteome Discoverer. We then used the area under the curve of each fragmented precursor ion, recorded by the software, as the measure of protein abundance. To ensure we did not eliminate low mass proteins with few possible tryptic peptides (e.g cyclophilin and ubiquitin) we included identities based on a single peptide provided that the MS/MS spectrum was of sufficient quality to guarantee a high confidence score. This reduced the data for analysis to 633 proteins at a false discovery rate of $1 \%$.

\section{Abundance of SWAP constituents is not evenly distributed}

An intensity plot of the recorded area score for each identified protein shows a markedly skewed distribution with a relatively small number of proteins being highly abundant and many present in only trace amounts (Fig. 1a). The dynamic range of modern proteomic analysis was revealed by the highest $(78,766)$ and lowest (3.6) values in the 633 protein series, which differed by more than four orders of magnitude. The proteins were arranged in descending order by abundance to record the cumulative total area, and the percentage contribution of each determined (Fig. 1b). Twenty percent of the total SWAP mass was contributed by only five proteins, namely enolase, aldolase, actin, glyceraldehyde 3-phosphate dehydrogenase and fatty acid-binding protein (Table 1). Fifty per cent of the total mass was represented by only 18 proteins and a further 149 made up $90 \%$ of the total. The remaining $10 \%$ was accounted for by 467 proteins each present in very small amounts. We chose to focus subsequent analyses on the 167 proteins that comprise the bulk of the mass up to the $90^{\text {th }}$ percentile. Proteomic analyses of other systems have revealed that the log concentration of protein abundance displays a normal distribution. Our SWAP preparation (Fig. 1c) appears visually skewed towards the more abundant components so we tested its normality using Shapiro-Wilk test proving a non-Gaussian distribution $(p=0.016)$.

\section{SWAP constituents are heavily biased towards cytosol and cytoskeleton}

We first searched the total dataset for matches to signature proteins identified by our previous proteomic investigations, color-coding the observed similarities. Sixteen 

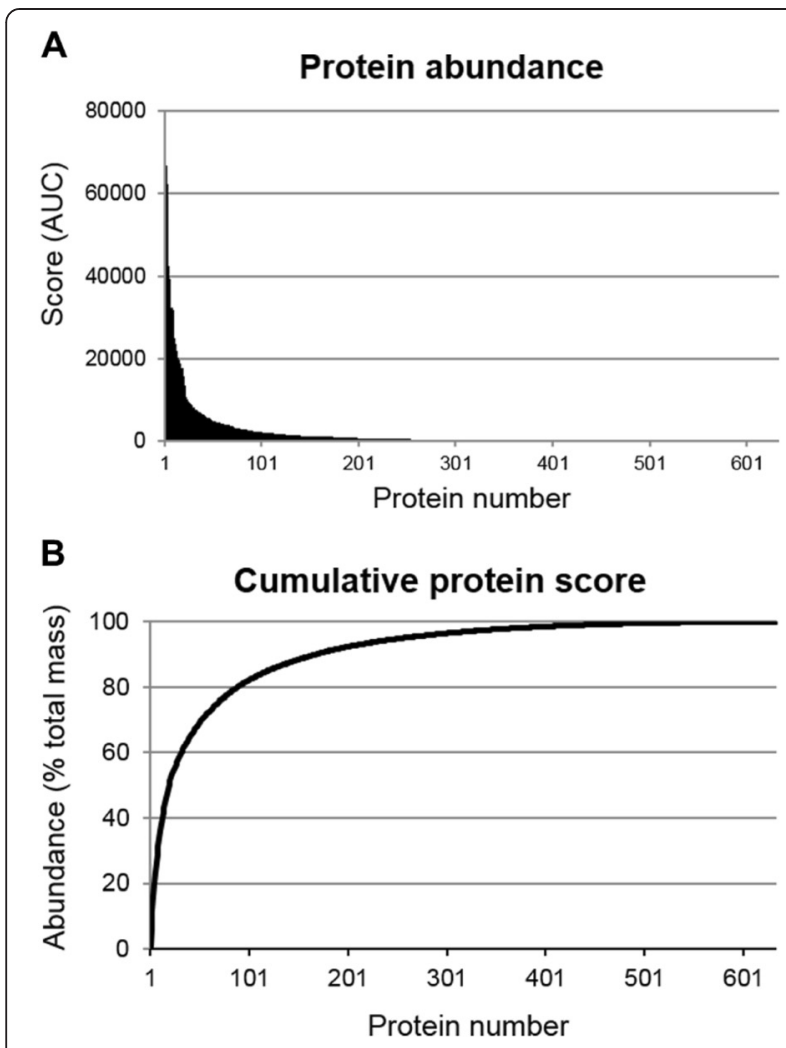

C

Frequency distribution of proteins in SWAP

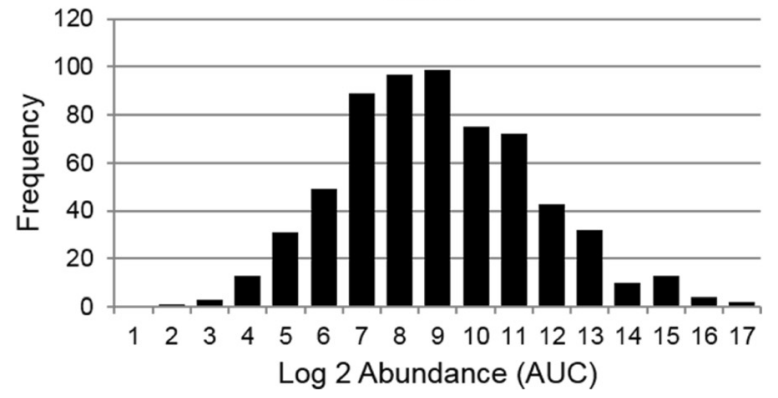

Fig 1 Protein distribution in SWAP. a Protein abundance of SWAP determined using the absolute Area Under Curve (AUC) values recorded for each of the 633 proteins identified. Note that a very small number of proteins contributed to the most prominent MS peaks detected. $\mathbf{b}$ Protein abundance in SWAP represented as a cumulative frequency plot revealed that only 18 constituents contributed to $50 \%$ of the total mass. c Frequency distribution plotted after $\log _{2}$ transformation of the calculated AUC for each protein. Although the distribution superficially appears Gaussian, a Shapiro-Wilk test revealed that it is not $(p<0.05)$, high abundance proteins being over-represented

out of 17 proteins identified by Curwen et al. 2004 [4] were equally prominent in our cytosolic fraction, many of them occupying the top positions in both studies. Approximately half the proteins identified as constituents of the worm vomitus were detected; these were almost exclusively the hydrolytic enzymes not the transporter proteins. Only seven out of 61 verified tegument surface proteins were present in SWAP, with three, Sm200, Sm29 and an annexin within the $90^{\text {th }}$ percentile. We then grouped each one of the 167 top proteins by subcellular or tissue location, by manual searching of several databases. Ten putative proteins were unusable or they were hypothetical, not yielding a meaningful annotation after BLAST searching of NCBInr, and were transferred to the residual unclassified category. These were approximately balanced by adding the known gut and tegument proteins in the bottom 467 identities. The color-coded proteins were then sorted by function/ tissue category and the total mass contributed by each group illustrated in a pie-chart (Fig. 2).

The largest category by far $(\sim 60 \%)$ comprises intracellular proteins from the cytosol. They function principally in glycolysis, protein translation, protein folding and the response to stress, all classical housekeeping activities. The second largest category (14\%) comprises proteins of the cytoskeleton and associated proteins, many of which will be present in the musculature. We classified calcium-binding proteins as a separate category (8\%) since they are found in many cellular locations and tissues like the tegument. Although mitochondria and nuclei are abundant cellular constituents, proteins originating therein comprised only 2.5 and $2 \%$ of the total, respectively. The few mitochondrial proteins appear to come from the matrix, likely released upon sonication (Additional file 1). The total mass contribution of tegument and gut proteins amounts to only $3 \%$. Further small contributions of protein are made by extracellular matrix, the secretory pathway and glycogen metabolism, this last representing parenchymal tissue.

\section{A small number of proteins comprise half the mass}

A detailed consideration of the top 18 proteins comprising half the total mass revealed that all were intracellular with only one (Smp_049550, $78 \mathrm{kDa}$ glucose regulated protein; Table 1$)$ possessing a signal peptide $(p=0.999)$. However, this protein is a Hsp-70 family member involved in protein folding and localized to the lumen of the endoplasmic reticulum. The remaining 17 proteins are all located in the cytosol rather than in an organelle and so unlikely to be $\mathrm{N}$ - or O- glycosylated for export (Table 1). When grouped by cellular function the five enzymes involved in energy metabolism (Table 1, EM) accounted for $39 \%$, and the four chaperones $(\mathrm{CH})$ for $22 \%$. The two cytoskeletal proteins (CY) comprise $13 \%$, the three calcium-binding proteins $10 \%$ and the two stress-response proteins (SR) $9 \%$. Fatty acid-binding protein, involved in intracellular lipid transport (ILT), made up the final $7 \%$. The abundance of all these proteins, apart from cyclophilin, is confirmed by the large number of diagnostic peptides (range 4-17, mean 8.4) 
Table 1 Top 18 most abundant proteins representing half of the total mass in SWAP

\begin{tabular}{|c|c|c|c|c|c|c|c|c|c|c|c|}
\hline Gene DB Accession & Description & Class & \# of Peptides & $\%$ Coverage & PD Area ${ }^{a}$ & $\mathrm{MW}[\mathrm{kDa}]$ & $\%$ total SWAP & Homo sapiens Accession & $\%$ Identity & $\%$ Cons. & Signal peptide \\
\hline Smp_024110.1 & Enolase & EM & 12 & 33,4 & 78,766 & 47,0 & 6,1 & NP_001419.1 & 75 & 86 & no \\
\hline Smp_042160.1 & Aldolase & EM & 11 & 27,2 & 66,915 & 35,4 & 5,2 & NP_005156.1 & 69 & 80 & no \\
\hline Smp_161930.1 & Actin & CY & 7 & 30,3 & 62,296 & 35,5 & 4,8 & gb|AAP37280.1 & 67 & 71 & no \\
\hline Smp_056970.1 & Glyceraldehyde $3 \mathrm{P}$ dehydrogenase & EM & 6 & 25,7 & 48,106 & 36,4 & 3,7 & NP_002037.2 & 73 & 82 & no \\
\hline Smp_095360.1 & Fatty acid-binding protein, Sm 14 & ILT & 4 & 39,9 & 42,434 & 14,8 & 3,3 & gb|AAB87141.1 & 47 & 61 & no \\
\hline Smp_054160.1 & Glutathione S-transferase, GST 28 & SR & 7 & 42,7 & 39,378 & 23,8 & 3,1 & AAA58623.1 & 30 & 48 & no \\
\hline Smp_106930.1 & Heat shock $70 \mathrm{kDa}$ protein & $\mathrm{CH}$ & 17 & 29,7 & 32,378 & 69,8 & 2,5 & NP_006588.1 & 84 & 92 & no \\
\hline Smp_050390.1 & Aldehyde dehydrogenase & EM & 11 & 31,4 & 32,212 & 53,7 & 2,5 & gb|AAH01619.1 & 57 & 72 & no \\
\hline Smp_009760.1 & 14-3-3 protein zeta/delta & $\mathrm{CH}$ & 6 & 24,6 & 31,822 & 28,4 & 2,5 & NP_003397.1 & 64 & 74 & no \\
\hline Smp_040130.1 & Cyclophilin & $\mathrm{CH}$ & 1 & 8,70 & 30,114 & 17,6 & 2,3 & AAH05982.1 & 67 & 73 & no \\
\hline Smp_034840.1 & 14-3-3 protein epsilon & $\mathrm{CH}$ & 11 & 48,0 & 24,916 & 26,0 & 1,9 & NP_006752.1 & 65 & 82 & no \\
\hline Smp_086330.1 & Calponin & $C B$ & 7 & 44,2 & 23,786 & 21,2 & 1,8 & XP_006722711.1 & 47 & 64 & no \\
\hline Smp_183710.1 & Actin & CY & 7 & 27,7 & 21,927 & 41,7 & 1,7 & NP_001092.1 & 96 & 99 & no \\
\hline Smp_049550.1 & 78 kDa glucose regulated protein & $\mathrm{CH}$ & 15 & 25,0 & 20,302 & 71,2 & 1,6 & NP_005338.1 & 75 & 87 & yes \\
\hline Smp_045200.1 & Tegument-allergen-like protein Sm22.6 & CB & 5 & 25,8 & 19,594 & 22,6 & 1,5 & $\mathrm{pdb}|2 \mathrm{PMY}| \mathrm{A}$ & 29 & 50 & no \\
\hline Smp_008070.1 & Thioredoxin & SR & 7 & 71,7 & 19,167 & 11,9 & 1,5 & NP_003320.2 & 47 & 61 & no \\
\hline Smp_086530.1 & Tegument-allergen-like protein Sm20.8 & $C B$ & 7 & 47,0 & 18,922 & 20,8 & 1,5 & BAD97069.1 & 22 & 49 & no \\
\hline \multirow[t]{2}{*}{ Smp_096760.1 } & Phosphoglycerate mutase & EM & 10 & 50,0 & 17,642 & 28,4 & 1,4 & NP_000281.2 & 57 & 71 & no \\
\hline & & Means & 8,4 & 35,2 & 35,038 & 33,7 & 2,7 & & 60 & 72 & \\
\hline
\end{tabular}

Annotations in italics were added by the authors

EM Energy metabolism, CY Cytoskeletal, ILT Intracellular lipid transport, SR Stress response, CH Chaperone, CB Calcium binding

${ }^{a}$ The Proteome Discoverer (PD) units of area under curve were divided by $10^{6}$ for ease of manipulation 


\section{SWAP}

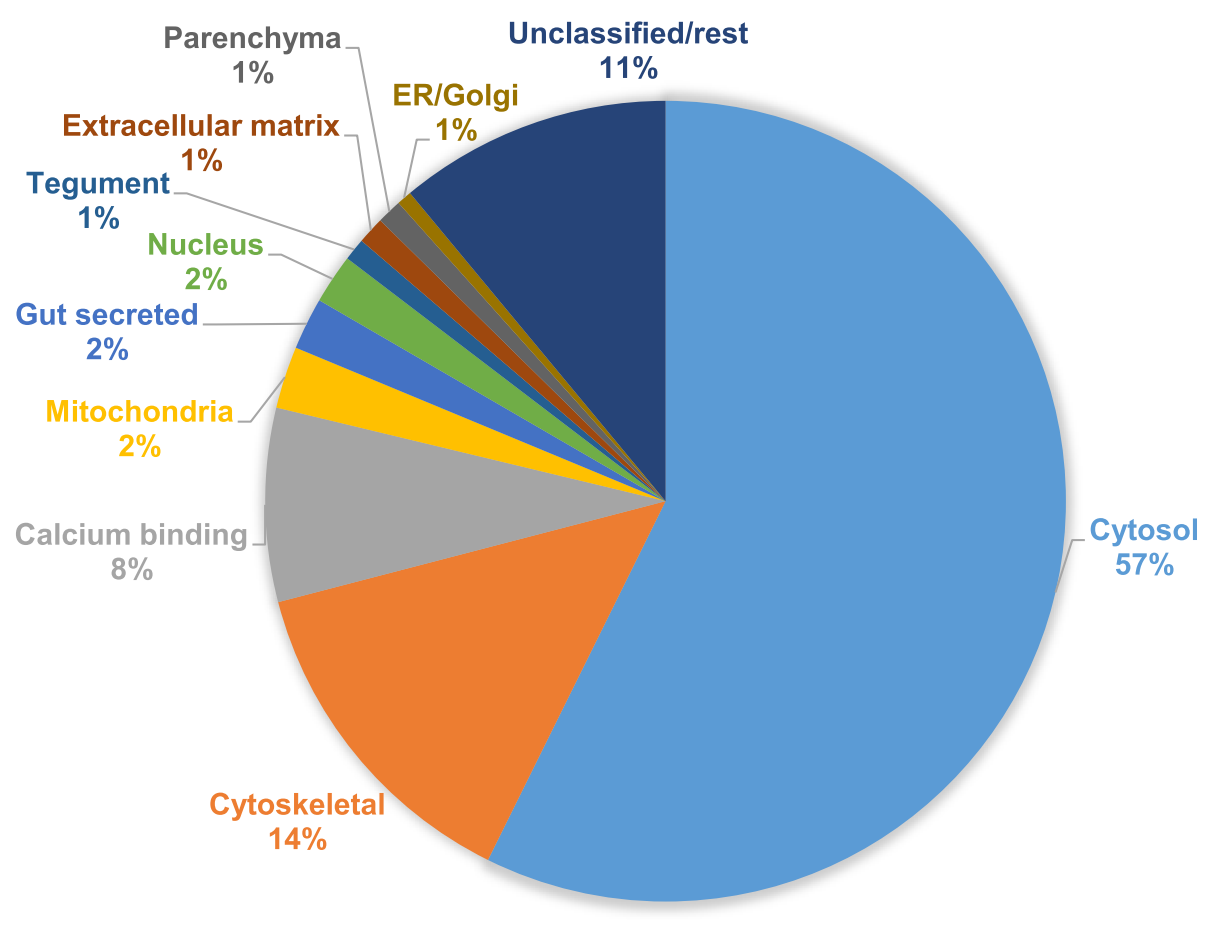

Fig 2 Sub-cellular or tissue location of SWAP constituents. Proteins within the $90^{\text {th }}$ percentile were categorized according to published literature, Uniprot and Blast searches. Soluble cytosolic and cytoskeletal proteins contribute to over $70 \%$ of total abundance in SWAP. Conversely, proteins proposed to function at host-parasite interfaces (e.g gut secreted and tegument surface) account for only $3 \%$ of the total mass

observed for each, amounting to a mean of $36.2 \%$ sequence coverage (range 9-72\%). All the top 18 identities are medium-sized proteins (range 12-71 kDa, mean $33.7 \mathrm{kDa})$. Fifteen out of 18 proteins have close homology in the human host (mean $66 \%$ ), the three exceptions being the EF-hand calcium-binding proteins Sm22.6 and Sm20.8 and glutathione S-transferase 28 (mean $27 \%$ ). It is notable that, at least three proteins in the first group and two in the second have been proposed as vaccine candidates.

\section{Discussion}

In this study we have provided the first inventory of SWAP constituents using a shotgun-based proteomic approach. The applied methodology combines highresolution liquid chromatography for peptide separation coupled to accurate $\mathrm{m} / \mathrm{z}$ detection of eluting molecules using a mass spectrometer. Compared to the classic 2D gel technique, shotgun analysis allows for detection of proteins with a wide dynamic range, differing in abundance by several orders of magnitude. In addition to protein identification, the shotgun strategy is also capable of estimating protein abundance through peak area detection of a precursor ion, using either tag-modified peptides [12], SILAC [13] or label-free methods [14, 15].
The last alternative requires minimal sample handling and can be applied to any detectable peptide, excluding the need for chemical tagging at specific amino acid residues. Here we have applied a stringent criterion for peptide selection, and hence protein identification, to gain insight into the relative abundance of the major constituents of the SWAP preparation. This approach increased significantly the repertoire of soluble worm protein constituents first described in 2004 using 2D gel electrophoresis [4].

Protein categorization revealed a preparation largely containing intracellular constituents that accounted for $97 \%$ of the identities. In the context of schistosome biology, this large fraction of proteins will only trigger immune effector mechanisms of the vertebrate host when parasite larvae, adult worms or eggs are damaged or die, releasing their cytosol into the host bloodstream or tissues. By extension, the protein constituents of SWAP are unlikely to be targets of a protective immune response because they are largely confined within the cells of live parasites. In stark contrast, proteins expressed at the host-parasite interfaces (namely the tegument and alimentary tract) are for the most part scarce or undetectable in SWAP accounting for only approximately $3 \%$ of the total mass. In the case of 
proposed tegument location, the only proteins detected were putative constituents of the membranocalyx namely Sm200, an annexin, CD59 and Sm29 [10, 11]. Concerning the last of these, its recorded relative abundance was too small to be included in the $90^{\text {th }}$ percentile of SWAP constituents. Annexin aside, the other three proteins Sm200, CD59 and Sm29 are known to be GPI-anchored [11], and their presence in SWAP could be explained by physical extraction of such membrane proteins during sample homogenization. Based on the aforementioned percentages it is clear that when SWAP is used to probe host cellular or antibody responses, the fraction of these attributable to surface-exposed or secreted proteins is negligible. It is noteworthy to mention we only considered secretion/excretion of proteins by means of the classical secretory pathway. Previous studies performed in vitro reported the production of vesicles by schistosomes $[16,17]$ and exosome-like particles being formed in the tegument of other parasitic helminths (Echinostoma caproni and Fasciola hepatica) [18]. However, compositional analysis of the $S$. mansoni culture's supernant and the lipidome of the host's blood plasma did not indicate the release of phospholipids known to be enriched at the parasite's tegument surface [19]. Identification of such lipids would argue in favour of genuinely secreted exosomes. Thus, to our understanding prediction of signal peptide remains the most convenient approach to anticipate protein secretion by $S$. mansoni.

We tacitly acknowledged this limitation of SWAP in our study of rhesus macaque self-cure responses [20] where we developed two specific antigen fractions, SASP (Stimulated Adult Worm Secreted Preparation) and TSP (Tegument Surface Preparation), to investigate reactivity to gut and tegument constituents, respectively, by Western blotting. At least two major conclusions could be drawn from these macaque experiments. First, only low burden animals exhibited high antibody titers against those antigens. A major fraction of the reactive spots could not be easily visualized using high sensitivity staining methods of the corresponding replica gels, suggesting that likely protective antibodies are targeting minor components of the preparations. Second, particularly for the tegument enriched preparation, the complexity of serum reactivity is inversely proportional to final worm burden. It follows that any similar studies wishing to investigate host responses to proteins expressed at or secreted from interfaces will have limited utility if SWAP is used as the only antigen source.

SWAP preparations have been used for vaccination experiments in past decades with variable results [21,22]. The most consistent results were obtained in an extensive series of experiments where $1 \mathrm{mg}$ of SWAP was administered intradermally to mice along with $5 \times 10^{6}$ units of BCG $[23,24]$. The BCG alone elicited no protection whilst
SWAP alone produced a small non-significant effect [25]. Paramyosin, a muscle protein was the major immunogen detected by antibodies from vaccinated animals although paradoxically this group of researchers concluded that the 40-50\% protection achieved was based on T cell-mediated mechanisms involving interferon gamma production by $\mathrm{T}$ cells and activation of macrophages, not humoral responses [25]. Paramyosin is \#46 in our SWAP preparation by abundance, equating to $0.4 \%$ of the total protein or $4.4 \mu \mathrm{g} / \mathrm{mg}$. This is exactly in the range of affinity-purified paramyosin subsequently used to achieve $\sim 40 \%$ protection in mice with BCG as adjuvant [26]. In this same publication the authors note that the SWAP-depleted of paramyosin still possessed protective properties. This leads us directly to the observation that many of the most abundant constituents of our SWAP from S. mansoni (or S. japonicum) have been administered to mice to induce protective immunity.

A search of the literature reveals that these candidates include aldolase (\#2), GAPDH (\#4), fatty acid-binding protein (\#5), GST-28 (\#6), 14-3-3 (\#9), calponin (\#12), Sm22.6 (\#15) and Sm20.8 (\#17) [27-34] with levels of protection ranging from 30 to $50 \%$. The respective abundance of these proteins in $1 \mathrm{mg}$ of our SWAP preparation is $52,37,33,31,25,18,15$ and $15 \mu$ g, collectively amounting to $226 \mu \mathrm{g}$. If we add in other proposed candidates such as $\mathrm{Cu} / \mathrm{Zn}$ superoxide dismutase [35] (\#21, $15.3 \mu \mathrm{g})$, myosin heavy chain [36] (\#32, $6 \mu \mathrm{g})$ and protein disulphide isomerase [37] (\#34,5.8 $\mu \mathrm{g})$ it is clear that at least a quarter of SWAP by mass comprises putative vaccine candidates. However, there is clearly no additive effect when all these candidates are administered together as the SWAP cocktail. Indeed, this is an odd and unexplained feature of the protection induced in mice by what are almost entirely cytosolic and cytoskeletal (i.e. internal) candidates. It seems each must be triggering the same mechanism to approximately the same degree but the outcome always hits a ceiling of approximately $40 \%$ protection whether the antigen is given alone or en masse via SWAP. We intend to address this conundrum in a separate publication.

The choice of SWAP to probe host responses is pragmatic because it is easy to prepare, the yields of soluble protein are high and due to the intrinsic immunogenicity of its major constituents, it virtually guarantees a strong response in assays. However, our data suggest that this last point is a trap for the experimenter. The principal components we have identified have been termed cryptic antigens [2] because they are only released when the adult worm dies. Proteomic analysis has revealed a similar mixture of soluble proteins in the eggs that are deposited and die in the tissues $[4,38]$ so that the host will be continuously restimulated by major SWAP constituents throughout the course of an infection. It is 
worth to bear in mind that SWAP may contain other classes of antigens not particularly dealt with in this shotgun approach. Anyhow, lipids and glycolipids are unlikely to be enriched in SWAP as no organic solvents (e.g methanol-chloroform) are used during its preparation. In addition, we cannot rule out the possibility that identification of glycoproteins has been made in this study. This can be achieved by identification and quantitation of non-glycosylated tryptic peptides.

Recently, the rate of evolution of schistosome proteins has been investigated using the $\mathrm{dN} / \mathrm{dS}$ ratio of nucleotide substitutions as the criterion of selection pressure, in the three major schistosome species that infect humans [39]. The study revealed that genes encoding exposed micro-exon gene (MEG) proteins and some venom allergen-like (VAL) proteins had the highest ratios, compared to genes encoding internal proteins and gastrodermal secretions. This applied even to those proteins at the tegument surface, apart from two surface vaccine candidates, $\mathrm{Sm} 29$ and the extrinsic portion of tetraspanin TSP-2, which also had high $\mathrm{dN} / \mathrm{dS}$ ratios. These data indicate that there is a definitive tendency in the Genus Schistosoma for truly exposed antigens to diversify due to selection pressure, whereas the cryptic antigens vary much less. A strong immune response is mounted against the cryptic antigens when they are encountered, but cannot provide effective protection. Thus, survival of a schistosome population, and adaptation to new hosts, requires an evolutionary response that modifies exposed epitopes to extend longevity and facilitate worm reproduction. Paradoxically, while estimation of the $\mathrm{dN} / \mathrm{dS}$ ratio provides an indicator of diversification, by extension it also reveals that the existing parasite population has been able to accommodate the hostile response. Our compositional analysis of SWAP, in which the highly modified MEGs and VALs proteins are barely detectable, suggests that its use to probe host responses to find vaccine candidates is almost certain to point the researcher in the wrong direction. Furthermore, where major SWAP components (or SWAP itself) have been tested in vaccination experiments the $\sim 40 \%$ protection observed has some rational explanation other than specific acquired immunity.

\section{Conclusion}

In this work we conducted a quantitative shotgun proteomic analysis of SWAP greatly expanding the list of its constituents. Our data have shown that at least $80 \%$ of the identified molecules are primarily of intracellular origin whereas only $3 \%$ represent signature proteins derived from the tegument and gastrodermis, the major host-parasite interfaces. This finding should allow immunologists to appreciate the dominant targets of the host cellular and antibody responses they are monitoring when SWAP is used as an antigenic preparation. This study finally poses the question as to why vaccination with SWAP, containing so many proposed vaccine candidates, has not shown additive or even synergistic effects on the induction of protection.

\section{Additional file}

Additional file 1: Protein/peptide mass spectrometry data and derived parameters.

\section{Competing interests}

The authors declare that they have no competing interests.

\section{Authors' contributions}

LXN designed and carried out experiments, analysed data and drafted the manuscript; ALS designed and carried out experiments; RAW and WCB conceived and designed experiments, analysed data and drafted the manuscript. All authors read and approved the final manuscript.

\section{Acknowledgments}

This work was supported by Special Visiting Researcher Program (Coordenação de Aperfeiçoamento de Pessoal de Nível Superior - CAPES) grant number 170/ 2012, Ministry of Education, Brazilian Federal Government. LXN is a recipient of a CAPES scholarship for the funding of his PhD in Biotechnology. Additional support was provided by FAPEMIG (Fundação de Amparo à Pesquisa do Estado de Minas Gerais), grant number APQ-00950-12. The authors also acknowledge Fundação Oswaldo Cruz (Centro de Pesquisas René Rachou, Belo Horizonte, Brasil) from which cercariae were obtained.

\section{Author details}

'Laboratório de Enzimologia e Proteômica, Instituto de Ciências Exatas e Biológicas, Universidade Federal de Ouro Preto, Ouro Preto, Minas Gerais, Brazil. 'Centre for Immunology \& Infection, Department of Biology, University of York, PO Box 373, York Y010 5YW, UK.

Received: 17 April 2015 Accepted: 8 June 2015

Published online: 19 June 2015

\section{References}

1. Colley DG, Cook JA, Freeman Jr GL, Bartholomew RK, Jordan P. Immune responses during human schistosomiasis mansoni. I. In vitro lymphocyte blastogenic responses to heterogeneous antigenic preparations from schistosome eggs, worms and cercariae. Int Arch Allergy Appl Immunol. 1977:53(5):420-33.

2. Wilson S, Jones FM, van Dam GJ, Corstjens PL, Riveau G, Fitzsimmons CM, et al. Human Schistosoma haematobium antifecundity immunity is dependent on transmission intensity and associated with immunoglobulin G1 to worm-derived antigens. J Infect Dis. 2014;210(12):2009-16.

3. Ludolf F, Patrocinio PR, Correa-Oliveira R, Gazzinelli A, Falcone FH, TeixeiraFerreira A, et al. Serological screening of the Schistosoma mansoni adult worm proteome. PLoS Negl Trop Dis. 2014;8(3):e2745.

4. Curwen RS, Ashton PD, Johnston DA, Wilson RA. The Schistosoma mansoni soluble proteome: a comparison across four life-cycle stages. Mol Biochem Parasitol. 2004;138(1):57-66.

5. Gilmore JM, Washburn MP. Advances in shotgun proteomics and the analysis of membrane proteomes. J Proteomics. 2010;73(11):2078-91.

6. Mann M, Kulak NA, Nagaraj N, Cox J. The coming age of complete, accurate, and ubiquitous proteomes. Mol Cell. 2013;49(4):583-90.

7. Nagaraj N, Alexander Kulak N, Cox J, Neuhauser N, Mayr K, Hoerning O, Vorm O, Mann M. System-wide perturbation analysis with nearly complete coverage of the yeast proteome by single-shot ultra HPLC runs on a bench top orbitrap. Mol Cell Proteomics. 2012; 11(3).

8. Hall SL, Braschi S, Truscott M, Mathieson W, Cesari IM, Wilson RA. Insights into blood feeding by schistosomes from a proteomic analysis of worm vomitus. Mol Biochem Parasitol. 2011;179(1):18-29.

9. Braschi S, Wilson RA. Proteins exposed at the adult schistosome surface revealed by biotinylation. Mol Cell Proteomics. 2006;5(2):347-56. 
10. Braschi S, Curwen RS, Ashton PD, Verjovski-Almeida S, Wilson A. The tegument surface membranes of the human blood parasite Schistosoma mansoni: a proteomic analysis after differential extraction. Proteomics. 2006;6(5):1471-82.

11. Castro-Borges W, Dowle A, Curwen RS, Thomas-Oates J, Wilson RA. Enzymatic shaving of the tegument surface of live schistosomes for proteomic analysis: a rational approach to select vaccine candidates. PLoS Negl Trop Dis. 2011;5(3):e993.

12. Ross PL, Huang YN, Marchese JN, Williamson B, Parker K, Hattan S, et al. Multiplexed protein quantitation in Saccharomyces cerevisiae using aminereactive isobaric tagging reagents. Mol Cell Proteomics. 2004;3(12):1154-69.

13. Ong S-E, Blagoev B, Kratchmarova I, Kristensen DB, Steen H, Pandey A, et al. Stable isotope labeling by amino acids in cell culture, SILAC, as a simple and accurate approach to expression proteomics. Mol Cell Proteomics. 2002;1(5):376-86

14. Wang G, Wu WW, Zeng W, Chou CL, Shen RF. Label-free protein quantification using LC-coupled ion trap or FT mass spectrometry: Reproducibility, linearity, and application with complex proteomes. J Proteome Res. 2006;5(5):1214-23.

15. Elliott MH, Smith DS, Parker CE, Borchers C. Current trends in quantitative proteomics. J Mass Spectrom. 2009:44(12):1637-60.

16. Kusel JR, Gazzinelli G, Colley DG, de Souza CP, Cordeiro MN. The formation of surface membrane vesicles from schistosomula of Schistosoma mansoni. Parasitology. 1984;89(Pt 3):483-94.

17. Wang L, Li Z, Shen J, Liu Z, Liang J, Wu X, et al. Exosome-like vesicles derived by Schistosoma japonicum adult worms mediates M1 type immune-activity of macrophage. Parasitol Res. 2015:114(5):1865-73.

18. Marcilla A, Trelis M, Cortes A, Sotillo J, Cantalapiedra F, Minguez MT, et al. Extracellular vesicles from parasitic helminths contain specific excretory/ secretory proteins and are internalized in intestinal host cells. PLoS One. 2012;7(9):e45974

19. Retra K, deWalick S, Schmitz M, Yazdanbakhsh M, Tielens AG, Brouwers JF, van Hellemond JJ. The tegumental surface membranes of Schistosoma mansoni are enriched in parasite-specific phospholipid species. Int J Parasitol. 2015.

20. Wilson RA, Langermans JA, van Dam GJ, Vervenne RA, Hall SL, Borges WC, et al. Elimination of Schistosoma mansoni adult worms by rhesus macaques: basis for a therapeutic vaccine? PLoS Negl Trop Dis. 2008;2(9):e290.

21. Sadun EH, Lin SS. Studies on the host parasite relationships to Schistosoma japonicum. IV. Resistance acquired by infection, by vaccination and by the injection of immune serum, in monkeys, rabbits and mice. J Parasitol. 1959:45:543-8.

22. Murrell KD, Dean DA, Stafford EE. Resistance to infection with Schistosoma mansoni after immunization with worm extracts or live cercariae: role of cytotoxic antibody in mice and guinea pigs. Am J Trop Med Hyg. 1975;24(6):955-62.

23. James SL, Pearce EJ, Sher A. Induction of protective immunity against Schistosoma mansoni by a non living vaccine. I Partial characterization of antigens recognized by antibodies from mice immunized with soluble schistosome extracts. J Immunol. 1985;134(5):3432-8

24. James SL. Induction of protective immunity against Schistosoma mansoni by a non-living vaccine. $V$ Effects of varying the immunization and infection schedule and site. Parasite Immunol. 1987;9(5):531-41.

25. James SL, Pearce EJ. The influence of adjuvant on induction of protective immunity by a non-living vaccine against schistosomiasis. J Immunol. 1988;140(8):2753-9.

26. Pearce EJ, James SL, Hieny S, Lanar DE, Sher A. Induction of protective immunity against Schistosoma mansoni by vaccination with schistosome paramyosin (Sm97), a nonsurface parasite antigen. Proc Natl Acad Sci U S A. 1988:85(15):5678-82.

27. Marques HH, Zouain CS, Torres CB, Oliveira JS, Alves JB, Goes AM. Protective effect and granuloma down-modulation promoted by RP44 antigen a fructose 1,6 bisphosphate aldolase of Schistosoma mansoni. Immunobiology. 2008;213(5):437-46.

28. Argiro LL, Kohlstadt SS, Henri SS, Dessein HH, Matabiau W, Paris PP, et al Identification of a candidate vaccine peptide on the $37 \mathrm{kDa}$ Schistosoma mansoni GAPDH. Vaccine. 2000;18(19):2039-48.

29. Tendler M, Brito CA, Vilar MM, Serra-Freire N, Diogo CM, Almeida MS, et al. A Schistosoma mansoni fatty acid-binding protein, Sm14, is the potential basis of a dual-purpose anti-helminth vaccine. Proc Natl Acad Sci U S A. 1996;93(1):269-73
30. Balloul JM, Grzych JM, Pierce RJ, Capron A. A purified 28,000 dalton protein from Schistosoma mansoni adult worms protects rats and mice against experimental schistosomiasis. J Immunol. 1987;138(10):3448-53.

31. Schechtman D, Tarrab-Hazdai R, Arnon R. The 14-3-3 protein as a vaccine candidate against schistosomiasis. Parasite Immunol. 2001;23(4):213-7.

32. Tang XN, Ji F, Jiang YX, Zhan XD, Li CP. Preliminary studies on protective immunity induced by recombinant protein of Schistosoma japonicum P14 gene and its mechanism against schistosome infection in mice. Zhongguo xue xi chong bing fang zhi za zhi = Chinese journal of schistosomiasis, control. 2013;25(4):375-8. 410.

33. Pacifico LG, Marinho FA, Fonseca CT, Barsante MM, Pinho V, Sales-Junior PA, et al. Schistosoma mansoni antigens modulate experimental allergic asthma in a murine model: a major role for CD4+ CD25+ Foxp3+ T cells independent of interleukin-10. Infect Immun. 2009;77(1):98-107.

34. Mohamed MM, Shalaby KA, LoVerde PT, Karim AM. Characterization of Sm20.8, a member of a family of schistosome tegumental antigens. Mol Biochem Parasitol. 1998;96(1-2):15-25.

35. Shalaby KA, Yin L, Thakur A, Christen L, Niles EG, LoVerde PT. Protection against Schistosoma mansoni utilizing DNA vaccination with genes encoding Cu/Zn cytosolic superoxide dismutase, signal peptide-containing superoxide dismutase and glutathione peroxidase enzymes. Vaccine. 2003;22(1):130-6

36. Soisson LM, Masterson CP, Tom TD, McNally MT, Lowell GH, Strand M. Induction of protective immunity in mice using a $62-k D a$ recombinant fragment of a Schistosoma mansoni surface antigen. J Immunol. 1992;149(11):3612-20.

37. Cao X, Hong Y, Zhang M, Han Y, Wu M, Wang X, et al. Cloning, expression and characterization of protein disulfide isomerase of Schistosoma japonicum. Exp Parasitol. 2014;146:43-51.

38. Mathieson W, Wilson RA. A comparative proteomic study of the undeveloped and developed Schistosoma mansoni egg and its contents: the miracidium, hatch fluid and secretions. Int J Parasitol. 2010;40(5):617-28.

39. Philippsen GS, Wilson RA, DeMarco R. Accelerated evolution of schistosome genes coding for proteins located at the host-parasite interface. Genome Biol Evol. 2015:7(2):431-43.

\section{Submit your next manuscript to BioMed Central and take full advantage of:}

- Convenient online submission

- Thorough peer review

- No space constraints or color figure charges

- Immediate publication on acceptance

- Inclusion in PubMed, CAS, Scopus and Google Scholar

- Research which is freely available for redistribution 